\title{
On the Bäcklund Transformation for the Gel'fand-Dickey Equations
}

\author{
Mark Adler* \\ Department of Mathematics, Brandeis University, Waltham, MA 02254, USA
}

\begin{abstract}
We study the Bäcklund transformation of the Gel'fand-Dickey equations, and in particular how the factorization of $n^{\text {th }}$ order differential operators leads to Lax type equations for first order operators, generalizing work of Adler and Moser [1]. In a similar fashion we study the Toda equations.
\end{abstract}

\section{Introduction}

In the study of the Korteweg-deVries (KdV) equation,

$$
q_{t}=3 q q_{x}-\frac{1}{2} q_{x x x}
$$

a Bäcklund transformation for (1.1) can be made to play an important role, as in [1]. As is well known, (1.1) can be rewritten in the Lax form

Factoring

$$
\begin{gathered}
\frac{d L}{d t}=[P, L], \quad L=L(q)=-D^{2}+q \\
P=P(q)=D^{3}-\frac{3}{4}(q D+D q), \quad D=\frac{\partial}{\partial x} .
\end{gathered}
$$

$$
L=A^{T} A, \quad A=D-v, \quad A^{T}=-D-v,
$$

we find $q=q(v)=v_{x}+v^{2}$, and the Bäcklund transformation for (1.1), $q(v) \mapsto q(-v)$, corresponds to reordering the factors of $L$, i.e.,

$$
L=A^{T} A \mapsto \tilde{L} \equiv A A^{T}, \quad q(v) \mapsto q(-v) .
$$

The crucial point is that the transformation $q(v) \mapsto q(-v)$ preserves (1.1). The best way to see that for our purposes is to observe that if $v$ satisfies the so-called

* Part of this work was done under the auspices of the SFB program of the Mathematical Institute of Bonn University, and the other part was done under NSF contract No. MCS 79-01998 
modified Korteweg-deVries equation

$$
v_{t}=-\frac{3}{2} v^{2} v_{x}+\frac{1}{4} v_{x x x}
$$

then $q(v)$ satisfies (1.1), and in fact (1.4) may be rewritten (see [1])

$$
\frac{d A}{d t}=B(q(-v)) \cdot A-A \cdot B(q(v))
$$

hence

$$
\frac{d A^{T}}{d t}=P(q(v)) \cdot A^{T}-A^{T} \cdot P(q(-v))
$$

which upon differentiating $L=A^{T} A$ immediately implies (1.2). On the other hand, if we differentiate $\tilde{L}=A A^{T}$ we get (1.2) with $L(q(v)) \mapsto L(q(-v)), P(q(v)) \mapsto P(q(-v))$, and so equivalently $q(-v)$ satisfies (1.1). Note that the fact that (1.4) is invariant under $v \mapsto-v$ reflects itself in the invariance of Eq. (1.1) under the Bäcklund transformation $q(v) \mapsto q(-v)$. On the level of operators (at least formally speaking), as $A$ moves in the equivalence class defined by

$$
A \mapsto U_{1}^{-1} A U_{2}, \quad U_{i} U_{i}^{T}=I, \quad i=1,2,
$$

$L, \tilde{L}$ move in their similarity class according to

$$
L=A^{T} A \mapsto U_{2}^{-1} L U_{2}, \quad \tilde{L}=A A^{T} \mapsto U_{1}^{-1} \tilde{L} U_{1} .
$$

At this point it is good to note that formally $L$ is similar to $\tilde{L}$; however, rigorously this may not be the case, and this transformation may be used to change the spectrum of $L$ (see [2]).

The above considerations were proven and used in [1] to very effectively study rational solutions of the $\mathrm{KdV}$, and in [2] to construct soliton solutions for the $\mathrm{KdV}$. The relation between (1.1) and (1.4) was first proven in [3], and the factorization of $L$ and its relation to (1.1) and (1.4) was first observed in [2].

Now Gel'fand-Dickey [4] have generalizations of (1.1), where $q$ is replaced by $Q=\left(q_{0}, q_{1}, \ldots, q_{n-2}\right)$, and we have instead of (1.1), a system of partial differential equations for $A$, and more to the point, (1.2) is maintained, only now with an $L$ of the form $L=D^{n}+q^{n-2} D^{n-2}+\ldots+q_{0}$, and $P=P(Q)$. Kuperschmidt and Wilson in a recent paper [5], have observed that there is a factorization of the general $L$, analogous to the one for $L=-D^{2}+q$, namely

$$
L=A_{n-1} \cdot A_{n-2} \cdot \ldots \cdot A_{0}, \quad A_{j}=D+\sum_{j=1}^{n-1} v_{i} \omega^{i j},
$$

with $\omega$ a primitive $n^{\text {th }}$ root of unity. They also observed that there is a system of partial differential equations analogous to (1.4) satisfied by $V=\left(v_{1}, v_{2}, \ldots, v_{n-1}\right)$, in the sense that if $V$ satisfies these equations then $Q=Q(V)$ will satisfy the appropriate generalization of (1.1). We show in this note that all the above discussed considerations of [1] generalize to the present situation, and in fact the proofs to be given here throw light on some of the mysterious proofs of that paper. In the last section we discuss the Bäcklund transformation of the Toda equations as Eq. (1.3) occurs in an unusual way in this situation which has not been reported, to the best of our knowledge, in the literature. 
Finally, this note was motivated by some observations [6] of Ehlers and Knörrer. They showed that if one worked with a finite-zone potential in the Korteweg-deVries equation, i.e., if $L$ commutes with some differential operator $K$ of degree $2 j+1$ and hence satisfies an equation of the form $Q(L, K)=0, Q(x, y)=y^{2}$ $-f(x), f(x)=x^{2 j+1}+c_{j} x^{2 j}+\ldots+c_{0}$, then the process of adding a soliton to $q$ changes the hyperelliptic curve $y^{2}-f(x)=0$ to a singular curve of the form $y^{2}=(x-s)^{2} f(x)$. Here $s$ is the soliton parameter. The considerations in this paper and [6] show that in the general finite zone potential case where $L=D^{n}$ $+q^{n-2} D^{n-2}+\ldots+q_{0}$, if the relevant curve is written $Q(x, y)=0$, i.e., $Q(L, K)=0$, it will be replaced in the soliton addition process by a curve of the form $Q(x, y / x-s)=0$, which in this context necessarily will be singular if $Q(x, y)=0$ is nonsingular. Finally I'd like to thank J. Moser for a useful discussion concerning this work.

\section{The General Bäcklund Transformation}

Before stating the results of this section we need some preliminaries. Given an $n^{\text {th }}$ order differential operator of the form

$$
\begin{gathered}
L=L(Q)=D^{n}+q_{n-2} D^{n-2}+\ldots+q_{0}, \\
Q=\left(q_{0}, q_{1}, \ldots, q_{n-2}\right), \quad D=\frac{\partial}{\partial x},
\end{gathered}
$$

$Q$ a $C^{\infty}$ function of the variable $x$, if we assign $D$ a degree of $1, q_{j}$ a degree $n-j$, then $L$ is homogeneous of degree $n$. Let $s$ be a positive integer relatively prime to $n$, and set $P=P(Q)=\left(L^{s / n}\right)_{+}=D^{s}+b_{s-1} D^{s-1}+\ldots+b_{0}$, the differential operator part of the pseudo-differential operator $L^{s / n}$ (see [7] for amplification). Then, as observed by Gel'fand-Dickey [4],

$$
\text { degree }[P, L]=n-2 \text {. }
$$

They used this observation to define the Lax equations

$$
\frac{d L}{d t}=[P, L]
$$

which are thus partial differential equations, the generalized Korteweg-deVries equation, for $Q$, of the form

$$
Q_{t}=X(Q), \text { i.e., } \frac{d q_{i}}{d t}=K_{i}\left(Q, D Q, \ldots, D^{m_{t}} Q\right), \quad i=0, \ldots, n-2,
$$

$K_{i}$ a polynomial in its arguments. In fact much more is true of $(2.4)$ as is reported in $[4,7]$.

We now factorize $L$ according to [5], where the theory of matrix circulants is used, but first we need some definitions. Given the function $V=V(x)=\left(v_{1}(x)\right.$, 
$\left.v_{2}, \ldots, v_{n-1}\right)$, for $j$ an integer, $\omega$ a primitive $n^{\text {th }}$ root of unity, set

$$
\left\{\begin{aligned}
\Omega \cdot V= & \left(\omega v_{1}, \omega^{2} v_{2}, \omega^{i} v_{i}, \ldots, \omega^{n-1} v_{n-1}\right), \\
& V_{j} \equiv \Omega^{j} \cdot V \equiv \Omega^{j-1} \cdot(\Omega \cdot v)=\left(\omega^{j} v_{1}, \ldots, \omega^{i j} v_{i}, \ldots, \omega^{(n-1) j}\right) \\
A= & A(V)=D+\sum_{i=1}^{n-1} v_{i}, \quad A_{j}=A\left(V_{j}\right), \\
& L_{j} \equiv A_{j-1} \cdot A_{j-2} \ldots A_{0} \cdot A_{n-1} \cdot A_{n-2} \ldots A_{j}, \\
& j=0,1, \ldots, n-1,
\end{aligned}\right\}
$$

and so $L_{j}$ is an $n^{\text {th }}$ order differential operator of the form (2.1), as the coefficient of $D^{n-1}, \sum_{i, j} v_{i} \omega^{i j}$, vanishes since $\sum_{j=0}^{n-1} \omega^{i j}=0$. In [5] it is shown that given $L(Q)$ in the form (2.1), there exists (at least formally) a $V$ such that in the notation (2.5), we have

$$
L(Q)=L_{0}=A_{n-1} \cdot A_{n-2} \cdot \ldots \cdot A_{0} .
$$

Since the $A_{i}$ 's are functions of $V$, we think of the above as a partial differential equation for $V, Q=Q(V)$, and by solving it, we have factored the operator $L$. For $n=2, Q=Q(V)=v_{x}+v^{2}$. We now define the degree of $v_{i}$ to be one, that of $\frac{\partial v_{i}}{\partial x}$ to be two, etc., and this is consistent with the degree of $Q$. Note that by (2.5), $L_{j}=L_{0}\left(Q\left(V_{j}\right)\right)$. We define $P_{j} \equiv P\left(Q\left(V_{j}\right)\right), j$ an integer and we now record

$$
L_{j}=L_{0}\left(Q\left(V_{j}\right)\right), \quad P_{j}=P\left(Q\left(V_{j}\right)\right) .
$$

We need also define the operator

$$
P_{j+1} \cdot A_{j}-A_{j} \cdot P_{j} \equiv B_{j}(V)=B_{0}\left(V_{j}\right), \quad j=0,1, \ldots, n-1 .
$$

Note the definition $A_{j}, P_{j}, L_{j}$ really makes sense for all integers $j$, and in fact since $\omega^{n}=1$, they are all $n$ periodic functions of $j$. Let us also define

$$
L_{j}^{\prime} \equiv \sum_{s=0}^{n-1}\left(A_{j-1} \cdot A_{j-2} \cdot \ldots \cdot A_{s+1} \cdot B_{s} \cdot A_{s-1} \cdot \ldots \cdot A_{j}\right) .
$$

We then have the following results.

\section{Lemma 1.}

$$
L_{j}^{\prime}=\left[P_{j}, L_{j}\right]
$$

and hence degree $\left(\left[P_{j}, L_{j}\right]\right)=n-2$.

Lemma 2. $B_{0}(V)$, hence $B_{j}(V)$ (see (2.7)), is a multiplication operator and so may be interpreted as a function of $V$ and its derivatives, and shall be. In addition we have

$$
\sum_{j=0}^{n-1} B_{j}(V)=0 \text {. }
$$

We define the modified generalized Korteweg-deVries equations as follows: Set

$$
\frac{d A_{j}}{d t}=B_{j}(V)=P_{j+1} \cdot A_{j}-A_{j} \cdot P_{j}, \quad j=0,1, \ldots, n-1 .
$$


Since $\frac{d A_{j}}{d t}=\sum_{i=1}^{n-1} \omega^{j i} \frac{d v_{i}}{d t}=B_{j}(V)$, we can only require (2.11) at first for $j=0,1, \ldots, n-2$, which since $\operatorname{det}\left[\omega^{j i}\right]_{i=1, \ldots, n-1} \neq 0$, defines uniquely the modified $\mathrm{KdV}$ equations $j=0, \ldots, n-2$

$$
\frac{d V}{d t}=Y(V)
$$

this is indeed equivalent to (2.11), for $\sum_{j=0}^{n-1} A_{j}=n D$, and (2.10), $\sum_{j=0}^{n-1} B_{j}(V)=0$, yields

$$
\frac{d A_{n-1}}{d t}=-\sum_{j=0}^{n-2} \frac{d A_{j}}{d t}=-\sum_{j=0}^{n-2} B_{j}(V)=B_{n-1}(V),
$$

and thus (2.11) for $j=n-1$ follows automatically from the cases $0 \leqq j \leqq n-2$.

We can now state the main result:

Theorem 1. The generalized modified Korteweg-deVries equations (2.12) are equivalent to the deformations

$$
\begin{aligned}
\frac{d A\left(\Omega^{j} \cdot V\right)}{d t}= & P\left(Q\left(\Omega^{j+1} \cdot V\right)\right) \cdot A\left(\Omega^{j} \cdot V\right)-A\left(\Omega^{j} \cdot V\right) \cdot P\left(Q\left(\Omega^{j} \cdot V\right)\right), \\
& 0 \leqq j \leqq n-1
\end{aligned}
$$

which moreover imply the Lax-equations

$$
\frac{d L\left(Q\left(\Omega^{j} \cdot V\right)\right)}{d t}=\left[P\left(Q\left(\Omega^{j} \cdot V\right)\right), L\left(Q\left(\Omega^{j} \cdot V\right)\right)\right], \quad j=0,1, \ldots, n-1,
$$

and hence setting $j=0,(2.12)$ implies the generalized Korteweg-deVries equations

$$
\frac{d Q}{d t}=X(Q)
$$

In addition, the Eq. (2.12) is invariant under the action

$$
\left.V \rightarrow \Omega \cdot V \quad \text { (and hence } V \rightarrow \Omega^{j} \cdot V\right),
$$

i.e., $\left\{\operatorname{diag}\left(\omega, \omega^{2}, \ldots, \omega^{(n-1)}\right)\right\}^{-1} Y(\Omega \cdot V)=Y(V)$.

Remark. Given the operator $L$ and any factorization

$$
L=D_{n-1} \cdot D_{n-2} \ldots D_{0}
$$

as $\left(D_{n-1}, \ldots, D_{0}\right)$ evolves through the equivalence class

$$
\begin{aligned}
\operatorname{diag}\left(D_{n-1}, D_{n-2}, \ldots, D_{0}\right) \\
\quad \mapsto \operatorname{diag}\left(U_{n}, U_{n-1}, \ldots, U_{1}\right) \cdot \operatorname{diag}\left(D_{n-1}, \ldots, D_{0}\right) \cdot\left(\operatorname{diag}\left(U_{n-1}, U_{n-2}, \ldots, U_{1}, U_{0}\right)\right)^{-1} \\
\quad=\operatorname{diag}\left(U_{n} D_{n-1} U_{n-1}^{-1}, \ldots, U_{j+1} D_{j} U_{j}^{-1}, \ldots, U_{1} D_{j} U_{0}^{-1}\right), \quad U_{n}=U_{0},
\end{aligned}
$$

$L$ evolves through its similarity class, $L \rightarrow U_{0} L U_{0}^{-1}$; and if we define $L_{j}$ with regard to the above factorization in the obvious fashion $L_{j} \mapsto U_{j} U_{j}^{-1}$. Now our 
theorem is an instance of this situation, where formally $U_{j}$ is defined by

$$
U_{j}(t=0)=I, \quad \frac{d U}{d t}=U P_{j} .
$$

This theorem generalizes the situation for the case $n=2$, as promised in the introduction.

All the proofs are straightforward computations.

Proof of Lemma 1. By (2.7) and (2.8) we get the telescoping sum

$$
\begin{aligned}
L_{j}^{\prime}= & \left(P_{j} L_{j}-A_{j-1} P_{j-1} A_{j-2} A_{j-3} \ldots A_{0} A_{1} \ldots A_{j}\right) \\
& +\left(A_{j-1} P_{j-1} A_{j-2} A_{j-3} \ldots A_{0} \ldots A_{j}-A_{j-1} A_{j-2} P_{j-2} A_{j-3} \ldots A_{0} \ldots A_{j}\right) \\
& +\therefore \\
& \left(A_{j-1} \ldots A_{0} \ldots A_{j+1} P_{j+1} A_{j}-L_{j} P_{j}\right) \\
= & P_{j} L_{j}-L_{j} P_{j}=\left[P_{j}, L_{j}\right] .
\end{aligned}
$$

Proof of Lemma 2. Write $B_{0}(V)=b(V) D^{s}+\ldots, s \geqq 0$. Then by (2.8) and (2.5),

$$
\begin{aligned}
L_{0}^{\prime}=\sum D^{n-\ell-1} b\left(V_{\ell}\right) D^{s} D^{\ell}+\ldots \\
=\left(\sum_{\ell=0}^{n-1} b\left(V_{\ell}\right)\right) D^{n-1+s}+\text { lower order terms. }
\end{aligned}
$$

But $L_{0}^{\prime}$ is an $n-2$ order operator by Lemma 1 , and since $s \geqq 0$, we must have $\sum_{\ell=0}^{n-1} b\left(V_{\ell}\right)=0$. If $s \geqq 1, B(V)=b(V) D^{s}+\hat{b}(V) D^{s-1}+\ldots$, and the coefficient of the $D^{n-2+s}$ term in $L_{0}^{\prime}$, which must be zero, is

$$
\sum_{\ell=0}^{n-1}(n-\ell-1) \frac{\partial b}{\partial x}\left(V_{\ell}\right)+\sum_{\ell=0}^{n-1} \hat{b}\left(V_{\ell}\right)+\sum_{m \neq \ell} b\left(V_{\ell}\right)\left(A_{m}-D\right)=0,
$$

and if we consider $L_{k}^{\prime}$ instead of $L_{0}^{\prime}$, that amounts to substituting $V \mapsto V_{k}$ and so we get the general identity

$$
\sum_{\ell=0}^{n-1}(n-\ell-1) \frac{\partial b}{\partial x}\left(V_{\ell+k}\right)+\sum_{\ell=0}^{n-1} \hat{b}\left(V_{\ell+k}\right)+\sum_{m \neq \ell} b\left(V_{\ell+k}\right)\left(A_{m+k}-D\right)=0,
$$

where we have used $\left(V_{k}\right)_{\ell}=V_{k+\ell}$, as follows from (2.5). Note that the last two terms on the left hand side of (2.14) are actually $k$ independent, while since $\sum_{\ell=0}^{n-1} b\left(V_{\ell}\right)=$ $\sum_{\ell=0}^{n-1} b\left(V_{\ell+k}\right)=0,(n-1) \cdot \sum_{\ell=0}^{n-1} \frac{\partial b}{\partial x}\left(V_{\ell+k}\right)=0$. We may conclude that $\sum_{\ell=0}^{n-1} \ell \frac{\partial b}{\partial x}\left(V_{\ell+k}\right)$ is $k$ independent, and thus by homogeneity considerations, so is $\sum_{\ell=0}^{n-1} \ell b_{1}\left(V_{\ell+k}\right)$, and in
particular we have

$$
\begin{aligned}
& b\left(V_{1}\right)+2 b\left(V_{2}\right)+\ldots+(n-1) b\left(V_{n-1}\right) \\
& =b\left(V_{2}\right)+2 b\left(V_{3}\right)+\ldots+(n-1) b\left(V_{0}\right) .
\end{aligned}
$$


This equation yields, upon subtraction and making use of $\sum_{i=0}^{n-1} b\left(V_{i}\right)=0$,

$$
\begin{aligned}
0 & =b\left(V_{1}\right)+b\left(V_{2}\right)+\ldots+b\left(V_{n-1}\right)-(n-1) b\left(V_{0}\right) \\
& =\sum_{i=0}^{n-1} b\left(V_{i}\right)+n b\left(V_{0}\right)=n b\left(V_{0}\right),
\end{aligned}
$$

and so $b\left(V_{0}\right)=0$, hence $b \equiv 0$ if $s \geqq 1$. This proves Lemma 2 .

Proof of Theorem 1. We have the equivalence of (2.12) and (2.13) by definition, given Lemma 2, which made all the definitions meaningful. Note that (2.12) and equivalently (2.13) imply the Lax equations of the theorem, and hence (2.4) follows from (2.9) and the identification of $L_{j}^{\prime}$ with $\frac{d L_{j}}{d t}$ as a consequence of (2.11). Observe that (2.11) by periodicity is the same as (2.11) for all $j$, which is obviously equivalent, due to periodicity, with the same statement with $\Omega \cdot V$ substituted for $V$. Since (2.11) is equivalent to (2.12) the same statement can be made for (2.12). This concludes the proof of Theorem 1.

\section{The Bäcklund Transformation for the Toda Equations}

We first study the periodic equations [10], where the theory is most analogous to the previous section, i.e., we study differential equations of the form

$$
\frac{d a_{i}}{d t}=a_{i}\left(b_{i+1}-b_{i}\right), \quad \frac{d b_{i}}{d t}=2\left(a_{i}^{2}-a_{i-1}^{2}\right), \quad a_{i+n} \equiv a_{i}, \quad b_{i+n} \equiv b_{i} .
$$

Equivalent to (3.1) is a Lax equation for infinite $n$-periodic matrices. Namely let $T$ stand for the shift operator on infinite vectors $y, x=\left(\ldots, x_{-1}, x_{0}, x_{1}, \ldots\right) \in R^{\infty}$,

$$
(T x)_{i}=x_{i+1},
$$

and define the multiplication operator $y$ by

$$
(y \cdot x)_{i} \equiv y_{i} x_{i} \quad \text { and } \quad y^{2} \equiv y \cdot y,
$$

and so for example $\left(y^{2} \cdot T^{j}(x)\right)_{i}=y_{i}^{2} x_{i+j}$. Then if $a, b$ are the infinite $n$-periodic vectors

$$
(a)_{i} \equiv a_{i}, \quad(b)_{i} \equiv b_{i},
$$

and if $L, P$ are the infinite $n$-periodic matrix operators

$$
L=T^{-1} a \cdot+b \cdot+a \cdot T, \quad P=-T^{-1} a \cdot+a \cdot T,
$$

we find the $n$-periodic Toda equations (3.1) to be equivalent to the Lax equations

$$
\frac{d L}{d t}=[P, L] \text {. }
$$

To verify this observe

$$
\begin{aligned}
\frac{d L}{d t}= & T^{-1} \frac{d a}{d t} \cdot+\frac{d b}{d t} \cdot+\frac{d a}{d t} \cdot T=\left(-T^{-1} a \cdot+a \cdot T\right)\left(T^{-1} a \cdot+b \cdot+a \cdot T\right) \\
& -\left(T^{-1} a \cdot+b \cdot+a \cdot T\right)\left(-T^{-1} a \cdot+a \cdot T\right) \\
= & T^{-1}(a \cdot T(b)-a \cdot b) \cdot+2\left(a^{2}-T^{-1}\left(a^{2}\right)\right) \cdot+(a \cdot T(b)-a \cdot b) \cdot T
\end{aligned}
$$


and upon equating similar terms we have $\frac{d a}{d t}=a \cdot T(b)-a \cdot b, \dot{b}=2\left(a^{2}-T^{-1}\left(a^{2}\right)\right.$, which is just Eq. (3.1) in vector notation. We could, by the Kac-Moody isomorphism of $n$-periodic infinite matrices with $n \times n$ matrices over an indeterminate, use finite matrices, as was done in [8], but we shall prefer to use the notation of infinite matrices, and the reader can consult [8] for the recipe to turn all the infinite matrices into finite ones.

The $N=2 n$-periodic Kac-van Moerbeke [13] equations, as is known, play the role of the "modified-Toda equations". These differential equations are of the form

$$
\frac{d \alpha_{i}}{d t}=\alpha_{i}\left(\alpha_{i+1}^{2}-\alpha_{i-1}^{2}\right), \quad \alpha_{i} \equiv \alpha_{i+N},
$$

and if $\alpha$ is the infinite vector such that $(\alpha)_{i}=\alpha_{i}$, we may, as is well-known [9], write (3.7) in the Lax form

$$
\frac{d \mathscr{L}}{d t}=[\mathscr{P}, \mathscr{L}], \quad \mathscr{L}=T^{-1} \alpha \cdot+\alpha \cdot T, \quad \mathscr{P}=-T^{-2}(\alpha \cdot T(\alpha)) \cdot+(\alpha \cdot T(\alpha)) \cdot T^{2},
$$

and from (3.7) we certainly have

$$
\begin{gathered}
\frac{d \mathscr{L}^{2}}{d t}=\left[\mathscr{P}, \mathscr{L}^{2}\right], \\
\mathscr{L}^{2}=T^{-2} \cdot(\alpha \cdot T(\alpha))+\left(\alpha^{2}+T^{-1}\left(\alpha^{2}\right)\right)+(\alpha \cdot T(\alpha)) \cdot T^{2} .
\end{gathered}
$$

As Moser observed [9] in the nonperiodic case, (3.8) leads to the Toda equations, and in fact it yields two Toda equations. If we reorder the ordered basis of $R^{\infty},\left\{\left(e_{i}\right)_{j}=\delta_{i j}\right\}_{i \in I} \mapsto\left\{\left[e_{2 i}\right]_{i \in I},\left[e_{2 i+1}\right]_{i \in I}\right\}$, and interpret this as a permutation transformation of $R^{\infty}$, this just amounts to acting on operators, and hence on (3.8), by conjugation with a permutation matrix $S$. We find that (3.8) then block diagonalizes, in fact if we define the infinite $N=2 n$ periodic vectors $\beta, \gamma$ by

$$
(\beta)_{j} \equiv \alpha_{2 j-1}, \quad(\gamma)_{j} \equiv \alpha_{2 j},
$$

then one easily computes

$$
\left\{\begin{array}{l}
S \mathscr{L}^{2} S^{-1}=L_{1} \oplus L_{2}, \quad S \mathscr{P} S^{-1}=P_{1} \oplus P_{2}, \\
L_{1}=T^{-1}(\beta \cdot \gamma) \cdot+\left(\beta^{2}+T^{-1}\left(\gamma^{2}\right)\right) \cdot+(\beta \cdot \gamma) \cdot T, \\
P_{1}=-T^{-1}(\beta \cdot \gamma) \cdot+(\beta \cdot \gamma) \cdot T, \\
L_{2}=T^{-1}(\gamma \cdot T(\beta)) \cdot+\left(\gamma^{2}+\beta^{2}\right) \cdot+(\gamma \cdot T(\beta)) \cdot T, \\
P_{2}=-T^{-1}(\gamma \cdot T(\beta))+(\gamma \cdot T(\beta)) \cdot T,
\end{array}\right\}
$$

and so (3.9) is equivalent to

$$
\frac{d L_{1}}{d t}=\left[P_{1}, L_{1}\right], \quad \frac{d L_{2}}{d t}=\left[P_{2}, L_{2}\right]
$$

Note $L_{1}, L_{2}, P_{1}, P_{2}$ are $n$-periodic infinite matrix operators. Observe that (3.11) is obviously a pair of equations of the form (3.6), (3.5), i.e., it is two Toda equations 
with the $b$ 's and $a$ 's of (3.5) being respectively

$$
\begin{gathered}
b^{(1)}=\beta^{2}+T^{-1}\left(\gamma^{2}\right), \quad a^{(1)}=\beta \cdot \gamma ; \\
b^{(2)}=\gamma^{2}+\beta^{2}, \quad a^{(2)}=\gamma \cdot T(\beta) .
\end{gathered}
$$

We view the $b^{(i)}=b^{(i)}(\alpha), a^{(i)}=a^{(i)}(\alpha), L_{i}=L_{i}(\alpha), P=P_{i}(\alpha), i=1,2$, as functions of $\alpha$, and in fact

$$
b^{(1)}(T(\alpha))=b^{(2)}(\alpha), \quad a^{(1)}(T(\alpha))=a^{(2)}(\alpha),
$$

hence

$$
L_{1}(T(\alpha))=L_{2}(\alpha), \quad P_{1}(T(\alpha))=P_{2}(\alpha) .
$$

Thus the $N=2 n$-periodic Kac-Moerbeke equations (3.7) for $\alpha$ imply the $n$-periodic Toda equation for the $b_{i}, a_{i}, i=1,2$, of (3.12). Now define the $n$-periodic matrix

$$
A=\beta \cdot \gamma \cdot T,
$$

then one verifies immediately from (3.10) that

$$
L_{1}=A^{\dagger} A, \quad L_{2}=A A^{\dagger} \text {. }^{\star}
$$

We remark that this type of factorization of a Jacobi matrix is standard in numerical analysis.

It is now clear that we should interpret the map

$$
\left(b^{(1)}, a^{(1)}, L_{1}\right) \mapsto\left(b^{(2)}, a^{(2)}, L_{2}\right), \quad \text { i.e., } \quad \alpha \rightarrow T(\alpha),
$$

as a Bäcklund transformation of the $n$-periodic Toda equations. This map is effected by the involution (up to basepoint) on $L_{1}, L_{2}$ achieved by the map $\alpha \mapsto T(\alpha)$, which obviously preserves the Kac-Moerbeke equations (3.7). The map $\alpha \mapsto T(\alpha)$ plays the role of the map $v \rightarrow-v$ in (1.4), the modified Korteweg-deVries equation. The factorization (3.15), and the relation between $L_{1}, L_{2}$ is the precise analog of (1.3), the Bäcklund transformation on the operator level for the Korteweg-deVries equation.

Finally we show that, as one would expect from the last section, the pair of Toda Lax equations (3.11) is equivalent to

$$
\dot{A}=P_{2} A-A P_{1}
$$

which thus must be equivalent to (3.7) and (3.8). To see this, observe that since $P_{1}^{\dagger}=-P_{1}, P_{2}^{\dagger}=-P_{2},(3.16)$ is equivalent to

$$
\dot{A}^{\dagger}=P_{1} A^{\dagger}-A^{\dagger} P_{2}
$$

but clearly by (3.15), (3.16) and (3.17) imply (3.11). Now given (3.11), (3.14), we must have

$$
\dot{A}^{\dagger} A+A^{\dagger} \dot{A}=\left[P_{1}, L_{1}\right], \quad \dot{A} A^{\dagger}+A \dot{A}^{\dagger}=\left[P_{2}, L_{2}\right],
$$

which may be regard as a linear system for the unknown $\dot{A}$. Since omit (3.16) is a solution to the inhomogeneous linear system (3.18), to show it is the only

$\star \quad \dagger$ means transpose 
solution, we must verify the related homogeneous system has only the trivial solution, i.e., we must show that

$$
\delta^{\dagger} A+A^{\dagger} \delta=0, \quad \delta A^{\dagger}+A \delta^{\dagger}=0
$$

implies $\delta=0$. Thinking of $\alpha$ as an indeterminate, we may formally compute $A^{-1}$, hence Eq. (3.19) implies respectively $\delta=-\left(A^{\dagger}\right)^{-1} \delta^{\dagger} A, \delta=-A \delta^{\dagger}\left(A^{\dagger}\right)^{-1}$, and so $A^{\dagger} A \delta^{\dagger}=\delta^{\dagger} A A^{\dagger}$. Set $\delta^{\dagger}=\varepsilon A^{-1}$ in the last equation, yielding

$$
A^{\dagger} A \varepsilon A^{-1}=\varepsilon A^{\dagger}, \quad \text { so } \quad\left[\varepsilon, A^{\dagger} A\right]=0 .
$$

This implies, remembering the correspondence between the infinite and finite matrices which enables us to use the spectral theory of finite matrices, that $\varepsilon=p\left(A^{\dagger} A\right)$, with $p(x)$ a real algebraic function. In the above we are still thinking of $\alpha$, hence $A$, as an indeterminate. Substituting $\delta^{\dagger}=\varepsilon A^{-1}$ into $\delta A^{\dagger}+A \delta^{\dagger}=0$, and using $\left[\varepsilon, A^{\dagger} A\right]=0$, yields :

$$
\left(A^{-1}\right)^{\dagger} \varepsilon^{\dagger} A^{\dagger}+A \varepsilon A^{-1}=0, \quad \text { so } 0=\varepsilon^{\dagger}+\left(A^{\dagger} A\right) \varepsilon\left(A^{\dagger} A\right)^{-1}=\varepsilon^{\dagger}+\varepsilon .
$$

Since $\varepsilon=p\left(A^{\dagger} A\right)$ and $\varepsilon^{\dagger}+\varepsilon=0$, we have $2 p\left(A^{\dagger} A\right)=0$, thus $\varepsilon=0$.

We have thus proven:

Theorem 2. The $N=2 n$-periodic Kac-van Moerbeke equations

$$
\left\{\begin{array}{l}
\frac{d \alpha_{i}}{d t}=\alpha_{i}\left(\alpha_{i+1}^{2}-\alpha_{i-1}^{2}\right), \quad \alpha_{i} \equiv \alpha_{i+2 n} \\
\frac{d \mathscr{L}}{d t}=[\mathscr{P}, \mathscr{L}], \quad \mathscr{L}=T^{-1} \alpha \cdot+\alpha \cdot T
\end{array}\right\}
$$

imply the n-periodic Toda equations

$$
\left\{\begin{array}{l}
\frac{d a_{i}}{d t}=a_{i}\left(b_{i+1}-b_{i}\right), \quad \frac{d b_{i}}{d t}=2\left(a_{i}^{2}-a_{i+1}^{2}\right), \quad a_{i+n} \equiv a_{i}, \quad b_{i+n} \equiv b_{i}, \\
\frac{d L}{d t}=[P, L], \quad L=T^{-1} a \cdot+b \cdot+a \cdot T,
\end{array}\right\}
$$

with the respective pair of $(b, a)$ 's being given by

$$
\begin{aligned}
& b^{(1)}=b^{(1)}(\alpha)=\beta^{2}+T^{-1}\left(\gamma^{2}\right), \quad a^{(1)}=\beta \cdot \gamma ; \\
& b^{(2)}=\gamma^{2}+\beta^{2}, \quad a^{(2)}=\gamma \cdot T(\beta),
\end{aligned}
$$

and the respective $L=L(\alpha)$ being

$$
L_{1}=A^{\dagger} A, \quad L_{2}=A A^{\dagger}, \quad A=\beta \cdot+\gamma \cdot T .
$$

Moreover, the Lax-equations for the $L_{i}, i=1,2$, are equivalent to the deformation equation

$$
\dot{A}=P_{2} A-A P_{1} .
$$

We thus may interpret the map

$$
\left(b^{(1)}, a^{(1)}, L_{1}\right)(\alpha) \rightarrow\left(b^{(2)}, a^{(2)}, L_{2}\right)(\alpha)
$$


as the Bäcklund transformation for (3.1), effected by the map

$$
\alpha \mapsto T(\alpha) .
$$

Remark 1. An analogous statement can be made for the nonperiodic Toda matrix, except here the Bäcklund transformation is not effected by so simple a map as $\alpha \mapsto T(\alpha)$. In addition, all the maps now become birational in $\alpha^{2}, b, a^{2}$, instead of just uni-rational and can be explicitly computed.

Remark 2. It would be interesting to see what the analogous factorization and hence Bäcklund theory would be for the $m$-band matrices of Mumford-van Moerbeke [11].

Remark 3. We note that the only really new facts in Theorem 2 are the statements concerning (3.15), (3.17), and even these may be known to some specialists; however, we feel that the close analogies in the cases of Sect. 2 and 3 warrant the insertion of Sect. 3.

\section{References}

1. Adler, M., Moser, J.: Commun. Math. Phys. 61, 1-30 (1978)

2. Deift, P.: Duke Math. J. 45, No. 2, 1978

3. Miura, R.M. : J. Math. Phys. 9, 1202-1204 (1968)

4. Dickey, L.A., Gel'fand, I. : Funkt. Anal. Jego Prilozh. 10, No. 4 (1976)

5. Kuperschmidt, B., Wilson, G.: Modifying Lax equations and the second Hamiltonian structure. Invent. Math. 62, 403-436 (1981)

6. Ehlers, F., Knörrer, H. : An algebra-geometric interpretation of the Bäcklund transformation for the Korteweg-deVries equation (to appear)

7. Adler, M.: Invent. Math. 50, 219-248 (1979)

8. Adler, M., van Moerbeke, P.: Adv. Math. 38, 318-379 (1980)

9. Moser, J.: Adv. Math. 16, 1-23 (1975)

10. van Moerbeke, P.: Invent. Math. 37, 45-81 (1976)

11. van Moerbeke, P., Mumford, D.: Acta Math. 143, 93-154 (1979)

12. Krichever, I.: Funct. Analy. Prilo. 12 (1), 76-78 (1978)

13. Kac, M., van Moerbeke, P.: Proc. Nat. Acad. Sci., USA 12 (4), 1627-1629 (1975)

Communicated by J. Moser

Received January 16, 1981 
